\title{
Construcción y Validación de la Escala RAU de Rendimiento Académico Universitario
}

\section{Construction and Validation of the University Academic Performance RAU Scale}

\author{
María de Lourdes Preciado-Serrano ${ }^{1}$, Mario Ángel-González ${ }^{2}$, Cecilia Colunga-Rodríguez ${ }^{3}$, \\ Julio César Vázquez-Colunga ${ }^{4}$, Mario Alberto Esparza-Zamora ${ }^{5}$, Claudia Liliana Vázquez-Juárez ${ }^{6}$ \\ y Marcelo Patricio Obando-Changuán ${ }^{7}$
}

\begin{abstract}
Resumen
Se valida la estructura del cuestionario Rendimiento Académico Universitario bajo el componente teórico de dos dimensiones: elementos objetivos y subjetivos. Participaron 556 estudiantes de psicología de la ciudad de Guadalajara, México. El análisis factorial (exploratorio y confirmatorio) mostró que el componente subjetivo de 20 ítems se constituye por tres dimensiones (aportación en las actividades académicas, dedicación al estudio y falta de organización de los recursos didácticos) y el componente objetivo de seis ítems por dos dimensiones (promedio de calificaciones y actividades de estudio). Los índices de ajuste del análisis factorial confirmatorio estuvieron dentro de los parámetros y la fiabilidad alpha de Cronbach en la escala subjetiva fue aceptable en dos de tres factores (F1 $\alpha=.792 ; \mathrm{F} 2 \alpha=.774)$ y moderada en el F3 $(\alpha=.542)$. El posible uso e implicaciones del instrumento en el ámbito educativo se discute a la luz de otras investigaciones.
\end{abstract}

Palabras clave: rendimiento académico, análisis factorial, educación superior, validez

\begin{abstract}
The structure of the University Academic Performance questionnaire is validated under the theoretical component of two dimensions: objective and subjective elements. 556 psychology students from the city of Guadalajara, Mexico participated. Factor analysis (exploratory and confirmatory) showed that the subjective component of 20 items is made up of three dimensions (contribution in academic activities, dedication to study and lack of organization of teaching resources) and the objective component of six items is composed of two dimensions (grade point average and study activities). The adjustment indices of the confirmatory factor analysis were within the parameters and Cronbach's alpha reliability in the subjective scale was acceptable in two of three factors (F1 $\alpha=.792 ; \mathrm{F} 2 \alpha=.774)$ and moderate in F3 $(\alpha=.542)$. The possible use and implications of the instrument in the educational field are discussed in the light of other research.
\end{abstract}

Keywords: academic performance, factor analysis, higher education, validity

\footnotetext{
${ }^{1}$ Psicóloga, Doctorado en Ciencias de la Salud en el Trabajo. Profesor Investigador, Centro Universitario de Ciencias de la Salud de la Universidad de Guadalajara. Calle Circuito Monarquía Sueca 151, Zapopan, Jalisco, México. C.P. 45130. Tel.: +521 10585200 ext. 33900. Correo: delourdes.preciado@academicos.udg.mx

${ }^{2}$ Doctorado en Psicología. Profesor Investigador, Centro Universitario de Tonalá. Calle San Lorenzo 381, Guadalajara Jalisco, México. C.P. 44750. Tel.: +521 3313208625. Correo: mario.angel@academicos.udg.mx

${ }^{3}$ Psicóloga, Doctorado en Ciencias de la Salud Pública. Profesor Investigador, Centro Universitario de Ciencias de la Salud de la Universidad de Guadalajara; Hospital de Pediatría, Instituto Mexicano del Seguro Social, Jalisco. Calle Pitágoras 292, Guadalajara Jalisco, México, C.P. 44790. Tel.: +521 3311769236. Correo: ccolungar@yahoo.com (autor para correspondencia)

${ }^{4}$ Doctorado en Psicología. Profesor Investigador del Centro Universitario de Tonalá de la Universidad de Guadalajara. Calle Retorno Santa Clara Sur 622, Fraccionamiento Misión San Francisco I, Tonalá Jalisco, México. C.P. 45416. Tel.: +521 3314329403 Correo: julio.vazquez@academico.udg.mx

${ }^{5}$ Maestría en Ciencias de la Salud de la Adolescencia y la Juventud. Profesor del Centro Universitario de Ciencias de la Salud de la Universidad de Guadalajara. Calle Fray Domingo Juncosa 678, Zapopan, Jalisco, México. C.P. 45200. Tel.: +521 10585200. Correo: ezma0380@gmail.com

${ }^{6}$ Doctorado en Psicología. Profesor Investigador del Centro Universitario de Tonalá de la Universidad de Guadalajara. Avenida Nuevo Periférico 555, Ejido San José Tateposco, C.P. 45425 Tonalá, Jal. Tel.: +521 333540 3020. Correo: claudia.vazquez5425@ academicos.udg.mx

${ }^{7}$ Doctor en Psicología, Académico de la Universidad de las Fuerzas Armadas (ESPE), Ecuador. Av. General Rumiñahui S/N y Ambato Sangolquí, Pichincha, Ecuador. Tel.: +539 99 6046454. Correo: mpobando@espe.edu.ec
}

Revista Iberoamericana de Diagnóstico y Evaluación - e Avaliação Psicológica. RIDEP · N60 · Vol.3 · 5-14 · 2021

ISSN: 1135-3848 print /2183-6051online 


\section{Introducción}

A partir de las últimas décadas del siglo XX, los temas relacionados con la Psicología Educativa, versan respecto a los entornos y a la actuación de los profesores, que se vinculan con el proceso de aprendizaje del estudiante. Los modelos educativos que enfatizan los estilos de enseñanza y aprendizaje, tienen la finalidad de orientar por medio de la planeación, operacionalización de secuencias didácticas y evaluaciones específicas, los contenidos de las materias para incidir en el rendimiento académico y en la satisfacción de los estudiantes (AlducinOchoa \& Vázquez-Martínez, 2017).

Además, el papel que juega la institución u organismo educativo es fundamental, ya que tiene que satisfacer las expectativas no sólo de los estudiantes, sino de la sociedad que los recibirá para el progreso, la producción o creación de fuentes de trabajo, además, debe cumplir con las exigencias de los financiadores de la educación (públicos o privados), que pueden coincidir o no con los objetivos educativos de los estudiantes, de los profesores o de la comunidad (Ruslin \& Jelas, 2010).

Por otra parte, los modelos que se centran en el estudiante, permiten validar componentes cognitivos, motivacionales y estilos de aprendizaje (Aragón \& Jiménez, 2009; Díaz, 2012; Molina, 2015; Inzunza et al., 2018). Sin embargo, uno de los aspectos más controversiales en la relación profesor-estudiante, es la evaluación del rendimiento académico de los pupilos, para lo cual algunos autores, han definido al rendimiento académico, como el logro, la construcción y ejecución del conocimiento de los estudiantes en los entornos educativos, cuyos indicadores pueden ser la expresión verbal, las actitudes y la práctica de valores (Avedaño et al., 2016).

Así, el registro de esos indicadores para valorar el rendimiento académico, puede ser a través de métodos cualitativos o cuantitativos, y también puede ser a través de los recursos didácticos previstos en el plan escolar de las materias o en las unidades de aprendizaje.

Algunos estudios reportan los beneficios del buen Rendimiento Académico (RA) de los estudiantes, relacionándolo con niveles altos de autoestima (Núñez \& Quinones, 2012) y con un adecuado auto-concepto (Salum-Farres et al., 2011). Otras investigaciones han referido al RA como resultado de estudiar en escuelas privadas y en el turno matutino (Ibarra \& Michalus, 2010; Lizasoain \& Joaristi, 2010). También, se han reportado hallazgos contradictorios del RA al relacionarlo con los estilos de aprendizaje activo, reflexivo, teórico o pragmático en estudiantes mexicanos con otras variables como la motivación (Ortiz-Fernández, et al., 2015; Ávalos, et al. 2018); con el semestre educativo o lugar de procedencia en estudiantes colombianos de Bogotá (Esguera \& Guerrero, 2010) y con el autoconcepto al inicio y final de los cursos educativos de estudiantes de educación media en Venezuela (Bolívar \& Rojas, 2014). El análisis de los antecedentes académicos y recibir tutorías desde los primeros semestres, se asocia significativamente con altos niveles de RA (García et al., 2012). Asimismo, se han reportado hallazgos que relacionan el RA con aspectos personales de los estudiantes, como su iniciativa personal en el ámbito educativo y su actitud emprendedora (Gorostiaga, et al., 2018).

Sin embargo, no existe un cuestionario que dimensione el constructo de rendimiento académico universitario (RAU) desde la perspectiva del estudiante, ya que por lo general se hace referencia a indicadores como el promedio de calificaciones o promedios de puntajes asignados por los profesores en las materias que se cursan (Alucín-Ochoa \& Vázquez-Martínez, 2017; Avedaño et al., 2016; Mayora-Pernía \& Fernández, 2015; Gómez et al., 2015). Además, si consideramos que diversos autores conciben al RA en torno al cumplimiento de metas, logros u objetivos establecidos de manera individual, determinados por la motivación o por la evaluación perceptual que hace la instancia educativa o el contexto social con el que se vinculan los estudiantes (Andrés et al., 2012; Caballero et al., 2007; Lamas, 2015; Reyes et al., 2016), se precisa contar con cuestionarios de autoinforme psicométricamente válidos para población mexicana, más allá del registro de calificaciones o logros escolares valorados por los docentes.

Desde este punto de vista, se elaboraron ítems a partir de la teoría socio-cognitiva (Zimmerman, 2013; Panadero \& Tapia, 2014), que ofrecen 
respuesta tipo Likert, para dimensionar los componentes objetivos y subjetivos del RA. Ambos componentes, objetivos y subjetivos, estarían correlacionados bajo el modelo de cinco variables endógenas. La Figura 1 muestra la estructura teórica del cuestionario, del lado izquierdo los ítems del componente subjetivo constituido con tres dimensiones: F1: aportación a las actividades académicas (que implica concentración en clases, gusto por exponer ante los compañeros los temas tratados, habilidades en la redacción de ensayos u otros textos, completar los estudios con cursos extraescolares, trabajar en equipo o con los compañeros entre otros elementos), F2: dedicación al estudio (que involucra la organización y el tiempo que se dedica a las actividades de estudio, planeación con anticipación la preparación de exámenes y la utilización adecuada de diversos recursos didácticos), y F3: falta de organización de los recursos didácticos (que contiene informes de las horas para dormir, carencia de recursos materiales para el estudio, olvido de contenidos temáticos, omisión en la entrega de deberes e inasistencias injustificadas). El componente objetivo se registra en dos factores: F4: Promedios de calificaciones (calificación actual, del último ciclo escolar universitario, del bachillerato/nivel anterior y de los cursos extras) y F5: calidad de estudio (horas de dedicación al día, libros y artículos académicos leídos en el último mes).

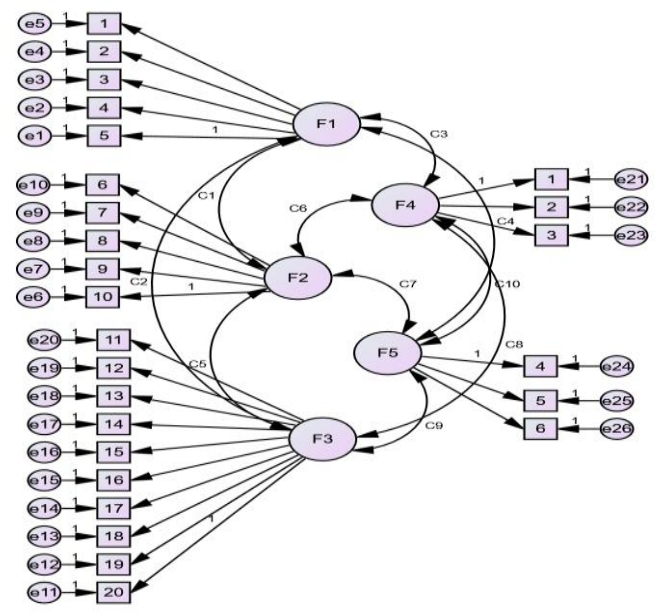

Figura 1. Modelo de relación de los componentes subjetivos (F1, F2, F3) y objetivos (F4, F5) de la escala de Rendimiento Académico Universitario. Se muestra la estructura correlacional y el error estadístico para cada ítem
Objetivo

Determinar la estructura factorial subyacente de la escala Rendimiento Académico Universitario (RAU) en estudiantes de la carrera de psicología en la ciudad de Guadalajara, México.

\section{Método}

\section{Participantes}

Se realizó un estudio transversal y analítico con la participación de una muestra intencional y no aleatoria de 556 estudiantes de la carrera de psicología, durante el año 2017, inscritos en tres universidades, una pública, de donde se obtuvo el $48 \%$ (264) de la muestra, y dos privadas de donde se recolectó el 52\% (292) del tamaño muestral. La selección fue mediante censo con la lista de asistencia de los profesores que imparten los cursos de segundo a octavo ciclo escolar, en ambos turnos, matutino y vespertino. Sin embargo, solo se encuestó a quienes voluntariamente lo desearon y cumpliendo con los criterios de inclusión, los cuáles consistieron en ser mayor de edad, alumnos con asistencia regular, que no estuvieran repitiendo materias previamente reprobadas, que no contaran con una carrera previa, ya sea terminada o inconclusa; se excluyeron del estudio a los estudiantes que por algún motivo, no aparecieran en las listas de asistencia oficiales. El 34\% (191) fueron hombres y el 66\% (365), mujeres. La edad promedio fue de 23 años con una $\mathrm{DE}=2.3$. El estado civil soltero registró al 98\% (544); casados al 1\% (8) y sólo cuatro estudiantes divorciados o separados. Tener hijos reportaron el 5\% (27) de los participantes y el 23\% (132) afirmó trabajar fuera del horario de clases. Cabe señalar, que al comparar las variables sociodemográficas de los estudiantes, no se encontraron diferencias estadísticas ( $p>.05)$.

\section{Instrumentos}

Se redactó una serie de enunciados fundamentados en la teoría socio-cognitiva de Zimmerman, (2013) que conformarían la escala $R A U$, los cuales se agruparon en dos aspectos: el primero con indicadores objetivos (6 ítems) que informan de los promedios de calificaciones (F4_1: del ciclo escolar previo, F4_2: del nivel anterior o bachillerato y F4_3: de cursos extras 
aprobados) y de las actividad académicas (F5_4: número de horas de estudio al día, F5_5: número de libros leídos en el último mes y F5_6: número de artículos académicos leídos en el último mes). El segundo aspecto consta de 20 proposiciones referidas a la autopercepción del rendimiento académico y se califica en una escala de siete puntos tipo Likert (0 nunca, 1 casi nunca, 2 algunas veces, 3 regularmente, 4 a menudo, 5 casi siempre, 6 siempre) con la finalidad de aplicar el análisis factorial exploratorio (Tabla 1). Además, se registraron datos socio-demográficos que permitieron describir las características de los participantes.

\section{Procedimiento}

Una vez que se redactó la versión final de la escala $R A U$, se aplicó a cinco docentes y a 10 estudiantes de nivel universitario, con el propósito de ajustar las palabras y asegurar que se comprendiera. Se hicieron las adecuaciones correspondientes al instrumento y posteriormente se solicitó a las autoridades académicas de una universidad pública y de dos universidades privadas, el ingreso para aplicar la prueba; cabe señalar que la muestra fue recabada por conveniencia, debido a lo cual los resultados no se refieren a una muestra nacional. Los estudiantes contestaron individualmente el cuestionario, pero se aplicó en grupos de 10 a 15, a fin de corroborar la auto-aplicabilidad. La participación fue de manera voluntaria y los datos se analizaron de manera confidencial, cumpliendo con los criterios éticos de investigación con seres humanos. El tiempo de respuesta fue de 10 a 30 minutos.

\section{Aspectos éticos}

El protocolo de investigación fue revisado y autorizado por un comité universitario de ética en investigación, perteneciente a la Coordinación de Investigación del Departamento de Salud Pública, de la Universidad de Guadalajara, el cual fue registrado con el código DSP/CI/02/17. La investigación se basó en los criterios éticos contemplados en la Ley General de Salud en México, referente a investigación en seres humanos, el cual se apega a normas y tratados internacionales. Para la aplicación de los instrumentos, se obtuvo el consentimiento informado por escrito, de todos los participantes y se respetó el derecho a la libre participación, así como el anonimato y la confidencialidad de los datos. No se ofreció ningún incentivo monetario o académico a los estudiantes por su participación en el estudio.

\section{Análisis de datos}

En primer término, se llevó a cabo el análisis con estadísticos descriptivos (media, desviación típica, asimetría y curtosis) para determinar la distribución normal de los ítems que conforman el segundo aspecto de la escala y después se efectuó el análisis factorial exploratorio (AFE) para determinar la estructura subyacente. Los datos se analizaron con el método de Componentes Principales bajo el principio de ortogonalidad y rotación de equamax, para minimizar el número de ítems y de factores que constituirían la escala final. Se determinó la hipótesis de diagonalidad de la matriz de correlación con el test de esfericidad de Bartlett al nivel de significancia $<.05$. Para identificar los efectos de colinealidad se utilizó la medida de adecuación de la muestra $\mathrm{KMO} \geq .75$ propuesta por Kaiser, Meyer y Olkin. Las dimensiones se conformaron con valor eigen superior a uno. Se determinó la matriz de covarianza para calcular los factores y se eligieron los ítems con cargas factoriales iguales o mayores a .30 para cada componente, dado que serían las primeras exploraciones del cuestionario.

Posteriormente, se llevó a cabo el análisis factorial confirmatorio (AFC) para corroborar la relación del componente subjetivo con el componente objetivo de la escala RAU, bajo el método de estimación de máxima verosimilitud a fin de comprobar si el ajuste con los valores cumplían con $X^{2} / d f$ (Chi-cuadrado/grados de libertad) en rango de 2 a 3 como adecuado, con los índices restrictivos (cuyas siglas son por sus nombres en inglés) de: GFI (índice de bondad de ajuste $\geq .9$ ), AGFI (índice ajustado de bondad de ajuste $>.8$ ) NFI (índice normado de ajuste $\geq .9$ ), CFI (índice de ajuste comparativo $\geq .9$ ), RMR (error cuadrático medio residual $\leq .08$ ), RMSEA (error de aproximación cuadrático medio <.08) y TLI (índice de no centralidad $\geq .95$ ) y el coeficiente de correlación múltiple superior a .5 para dejar las variables superficiales o ítems de la escala, según los valores sugeridos por Catena et al. (2003). Adicionalmente, se estimó la consistencia interna mediante el alpha de Cronbach $(\alpha)$ para las 
Tabla 1. Análisis factorial exploratorio y fiabilidad de la escala Rendimiento Académico Universitario componente subjetivo

\begin{tabular}{|c|c|c|c|}
\hline Ítem & Factor 1 & Factor 2 & Factor 3 \\
\hline 1. Dedico tiempo diario para realizar mis tareas que me asignan en mi carrera profesional. & & .751 & \\
\hline 2. Me organizo para ser eficiente en todas las actividades académicas. & & .749 & \\
\hline 3. Estudio con anticipación para presentar exámenes de conocimientos académicos. & & .672 & \\
\hline 4. Mis profesores emiten comentarios favorables de mi rendimiento académico. & .513 & & \\
\hline $\begin{array}{l}\text { 5. Utilizo diversos recursos didácticos (mapas conceptuales, diagramas, esquemas, etc.) } \\
\text { para aprobar las asignaturas de mi profesión. }\end{array}$ & & .654 & \\
\hline 6. Duermo menos de cinco horas, por estudiar un día antes de mis exámenes académicos. & & & .639 \\
\hline 7. Me ha faltado material adecuado para acreditar exámenes de los cursos. & & & .765 \\
\hline 8. He faltado a clase sin motivos importantes. & & & .507 \\
\hline $\begin{array}{l}\text { 9. Me olvido de entregar a tiempo los deberes (tareas o trabajos) que me asignan los } \\
\text { profesores. }\end{array}$ & & & .357 \\
\hline 10. Se me olvidan los contenidos de las asignaturas que ya he aprobado. & & & .390 \\
\hline 11. Estoy concentrado durante todas las asignaturas. & .437 & & \\
\hline 12. Me gusta exponer ante mis compañeros, los temas de las asignaturas. & .664 & & \\
\hline 13. Me considero que redacto bien ensayos o trabajos académicos. & .645 & & \\
\hline 14. Suelo completar mis estudios con cursos extra-académicos. & .496 & & \\
\hline 15. Me gusta estudiar material adicional al básico de las asignaturas. & .572 & & \\
\hline 16. Me es fácil trabajar en equipo para presentar mis deberes de las asignaturas. & .512 & & \\
\hline 17. Se aceptan mis ideas innovadoras cuando trabajo en equipo. & .586 & & \\
\hline 18. Yo participo activamente conforme con el contenido de las clases. & .662 & & \\
\hline 19. Mis calificaciones y evaluaciones son acorde con mi desempeño académico. & & .432 & \\
\hline 20. Estoy satisfecho con la carga de trabajo de las asignaturas & .484 & & \\
\hline Alpha de Cronbach & .792 & .774 & .542 \\
\hline
\end{tabular}

dimensiones que se obtuvieron en el componente subjetivo de la escala, en virtud de los valores continuos. Los datos se codificaron y analizaron con el programa Statistical Package for Social Sciences (SPSS) versión 21.

\section{Resultados}

\section{Análisis factorial exploratorio}

Los 20 ítems que conforman el segundo aspecto de la escala RAU mostraron distribución normal, por lo que se procedió a realizar el análisis factorial exploratorio aceptando valores eigen por arriba de .30 acorde con la condición muestral que se obtuvo (Lloret-Segura, FerreresTraver, Hernández-Baeza, \& Tomás-Marco, 2014) y a la congruencia teórica de su construcción. El indicador de esfericidad de Bartlett $(p<.05)$ comprobó la adecuación de la muestra para los 20 ítems. El índice KMO fue adecuado (.827). El análisis de varianza indicó que el $43.54 \%$ explica la extracción de tres componentes principales $(24.56 \%, 11.15 \%$ y $7.83 \%$ respectivamente).

Una vez analizada la estructura factorial del cuestionario RAU se procedió a identificar el contenido de los componentes a fin de nominar las variables subyacentes obtenidas y determinar la fiabilidad de cada una. Al primer factor, se le denominó aportación en las actividades académicas dado el contenido de los ítems que lo constituyen $(11,12,13,14,15,17,16,17,18,20)$; asimismo, el factor 2 se nominó como dedicación al estudio $(1,2,3,5,19)$, y el factor 3, se le llamó falta de organización de los recursos didácticos $(6,7,8,9,10)$. Estos ítems teóricamente están construidos de forma negativa al concepto y este análisis factorial sostiene su agrupación.

La Tabla 1 muestra las cargas factoriales de los componentes rotados después de cinco iteraciones y la consistencia interna con el coeficiente alpha de Cronbach. Los resultados mostraron que en dos de los tres factores, se encontró una consistencia interna aceptable (F1 $\alpha=.792 ; \mathrm{F} 2 \alpha=.774)$ y en el Factor 3 , una consistencia interna de $\alpha=.542$, considerada como moderada (Tabla 1).

\section{Análisis factorial confirmatorio}

En la Tabla 2, se presentan los índices aceptables para el análisis factorial confirmatorio (Ruiz et al., 2010) y los valores que se obtuvieron con la re-especificación de co-relaciones entre las variables superficiales propuestos por el modelo ofrecido. Los índices de bondad de ajuste del modelo propuesto fueron adecuados $\left(X^{2} / d f\right.$ : $797.65 / 261=3$ ), con un valor $p<.01$ significativo. 
Tabla 2. Índices de ajuste en el análisis factorial confirmatorio para la Escala Rendimiento Académico Universitario

\begin{tabular}{lcc}
\hline Estadístico & Valor aceptable & Índice obtenido \\
\hline$X^{2}$ & Ho & 797.658 \\
$D f$ & parámetros & 261 \\
$X^{2} / d f$ & $<3$ & 3 \\
$P$ & $<.05$ & .93 \\
GFI & $\geq .95$ & .90 \\
$A G F I$ & $>.95$ & .85 \\
NFI & $\geq .95$ & .93 \\
CFI & $\geq .95$ & .16 \\
RMR & cercano a cero & .06 \\
RMSEA & $<.08$ & .83 \\
TLI & $\geq .95$ & $1.092(1.61-1.392)$ \\
ECVI & Cercano a la unidad & \\
Nota. N=556. $X^{2}=$ Chi-cuadrado, $d f=$ grados de libertad, $p=$ significancia, GFI=índice de bondad de ajuste, AGFI=índice ajustado de bondad de \\
ajuste, NFI=índice normado de ajuste, CFI=índice de ajuste comparativo, RMR=error cuadrático medio residual, RMSEA=error de \\
aproximación cuadrático medio, TLI=índice de no centralidad. ECVI=índice de validación cruzada esperada (límite inferior-límite superior).
\end{tabular}

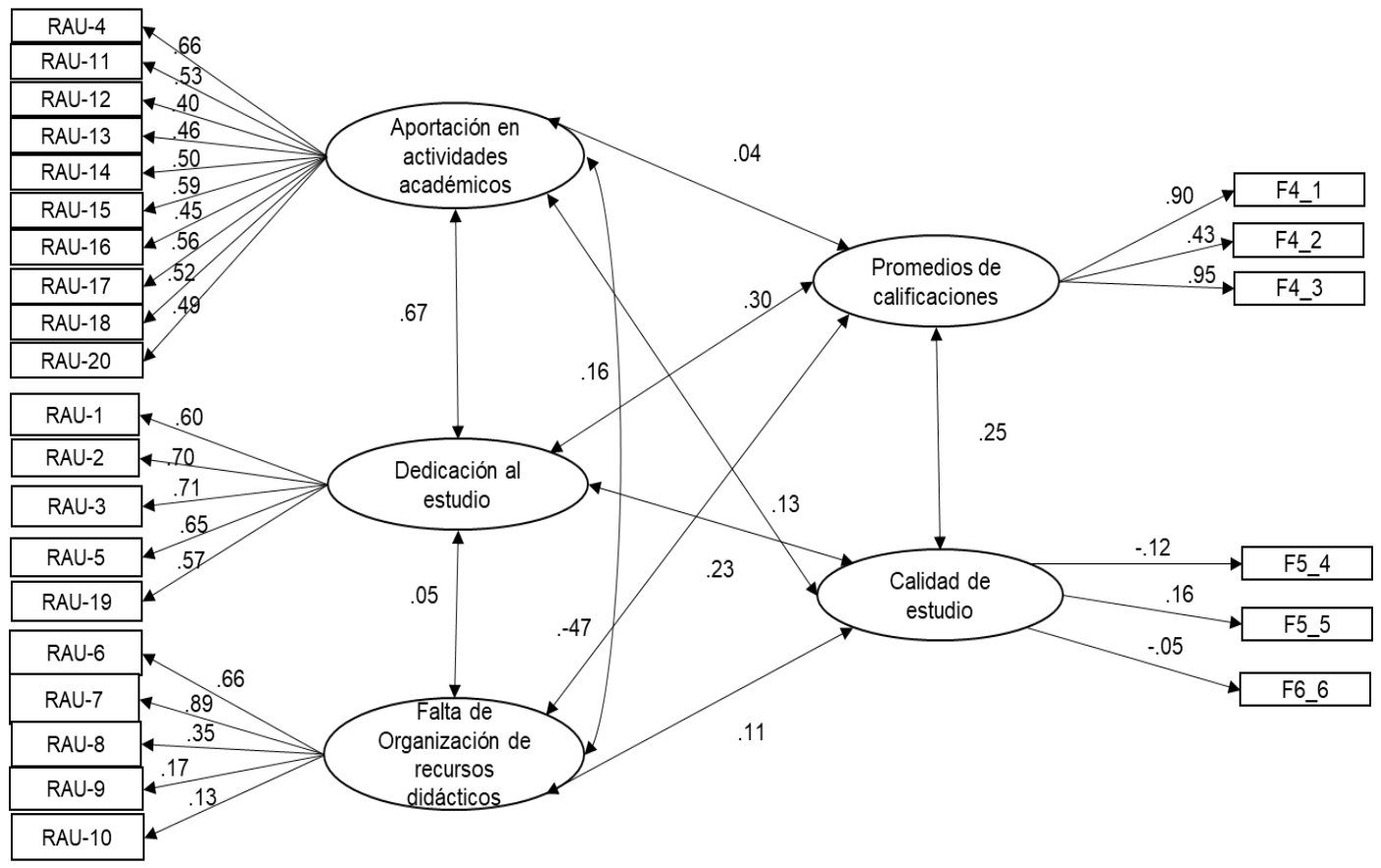

Figura 2. Modelo estructural factorial de la escala rendimiento académico universitario con los coeficientes estandarizados

Las correlaciones entre los errores de medida de cada ítem se ajustaron para maximizar la variabilidad. El índice de no centralidad (TLI=.83) reportó una ligera disminución en la proporción de valores esperados para estimar el modelo. Sin embargo, el índice de validación cruzada esperada, (ECVI) fue adecuado, lo que garantiza que las observaciones son independientes y el modelo generado es válido.

Analizando los coeficientes estandarizados de correlación para cada una de las vías propuestas (Figura 2), se observó que el Factor 2: dedicación al estudio correlacionó de forma baja pero significativa con el Factor 4: de promedios de calificaciones $(\mathrm{r}=.30, p<.01)$ y el Factor 3 : falta de organización de los recursos didácticos mantuvo correlación inversa con Factor 4: de promedios de calificaciones ( $\mathrm{r}=-.47, p<.01)$. En el análisis factorial confirmatorio se reportó correlación fuerte entre las dos primeras dimensiones del componente subjetivo, sin embargo, en todas las demás correlaciones significativas, se encontraron valores moderados y débiles. Cabe hacer notar que contrario a lo esperado por el supuesto teórico, la dimensión calidad de estudio, no mantiene correlación con las dimensiones del modelo, no obstante que si se elimina, los valores de validez disminuyen.

Con la finalidad de que el cuestionario Rendimiento Académico Universitario (RAU) pueda 
Tabla 3. Datos descriptivos de las dimensiones del cuestionario Rendimiento Académico Universitario

\begin{tabular}{lccccccccc}
\hline & Media & D.E. & Asimetría & $\begin{array}{c}\text { Error típico de } \\
\text { asimetría }\end{array}$ & Curtosis & $\begin{array}{c}\text { Error típico } \\
\text { de curtosis }\end{array}$ & bajo & medio & alto \\
\hline $\begin{array}{l}\text { Aportación a las actividades } \\
\text { académicas }\end{array}$ & 31.16 & 8.210 & -.241 & .104 & -.175 & .207 & 23 & $24-38$ & 39 \\
$\begin{array}{l}\text { Dedicación al estudio } \\
\begin{array}{l}\text { Falta de organización de los } \\
\text { recursos didácticos }\end{array}\end{array}$ & 22.03 & 5.030 & -.856 & .104 & .707 & .207 & 17 & $18-26$ & 27 \\
\hline
\end{tabular}

Nota. $\mathrm{N}=556$. D.E.=Desviación Estándar.

ofrecer un grado de valoración, se ofrecen los valores descriptivos en la Tabla 3 en este grupo de referencia; así el nivel bajo correspondería a la diferencia de una desviación estándar respecto de la media y el nivel alto con la suma correspondiente.

\section{Discusión}

Los resultados de esta investigación mostraron la validez del cuestionario Rendimiento Académico Universitario (RAU) diseñado para población mexicana en el contexto universitario de la ciudad de Guadalajara, bajo la acepción de dos dimensiones: componente objetivo (mediante el registro de promedios de calificaciones y actividades académicas extra aula) y componente subjetivo (enunciados con respuesta escalar). El análisis factorial confirmatorio determinó que la dimensión subjetiva se constituye con tres subescalas, dos en orden positivo (dedicación al estudio y aportación en las actividades académicas) y una en orden negativo (falta de organización de los recursos didácticos). Las dos subescalas positivas mostraron relación directa significativa con los promedios de calificaciones, en tanto que el componente negativo mantiene relación inversa y significativa.

Otros estudios han demostrado que las actividades académicas realizadas fuera de la escuela, como la dedicación de tiempo anticipado al estudio y la preparación de exámenes, así como la utilización de diversos recursos didácticos, favorecen el promedio de calificaciones. Por ejemplo, Reyes et al. (2016) en un análisis de regresión múltiple identificaron que factores personales como estudiar con amigos, utilizar enciclopedia, planear actividades con anticipación, entre otros, explican moderadamente la relación con altos puntajes en los exámenes EXANI-I y EXANI-II que aplica la Secretaría de Educación Pública en México como proceso de selección para ingresar al bachillerato. Asimismo, en este estudio indicaron que el factor escolar, como la procedencia de escuelas privadas, profesores que dejan tareas, cantidad de tareas, tareas entregadas y exámenes departamentales, explican aunque débilmente el rendimiento académico. Por tanto, contar con indicadores que valoren tanto subjetivamente como objetivamente la dedicación al estudio, como el tiempo invertido, la cantidad de material estudiado, el uso de técnicas de estudio, entre otros, puede monitorear el desempeño del estudiante.

Broc (2011) en su análisis de rendimiento académico en estudiantes de psicología identificó que el buen rendimiento se relaciona con la gestión del tiempo y los mecanismos que aplican para la reducción de estrés. Sin embargo, su acepción teórica sustenta que las variables volitivas y las relativas al aprendizaje son variables metacognitivas mediadoras del buen rendimiento académico y que predominantemente el realizar exámenes parciales y prácticas o ensayos previos se relacionan directamente con altos puntajes de rendimiento académico. Por tanto, es probable que la escala RAU que incluye ítems de acciones sistematizadas para el estudio, como las de organizar las actividades académicas, estudiar anticipadamente y completar su educación formal con acciones extra aula, permita con su aplicación realizar diagnósticos oportunos para apoyar adecuadamente a quienes se encuentren en situaciones vulnerables.

Chacón et al. (2017) reportan relación directa entre el auto concepto académico y el rendimiento académico en estudiantes universitarios deportistas, valorando la percepción que éstos tienen respecto a su rol a partir de los comentarios de sus profesores y de su concepción como inteligente o buen estudiante. Sin embargo, el autoconcepto familiar es un elemento que marca la diferencia entre quienes tienen éxito académico 
$\mathrm{y}$ quienes presentan bajos puntajes de calificaciones. Aunque, la escala RAU no valora los aspectos familiares, sí enfatiza en la organización de actividades extra-académicas, lo cual pudiera involucrar las habilidades para equilibrar las exigencias escolares y las necesidades familiares y sociales. Principalmente, en el género femenino, como lo indican AlducinOchoa y Vázquez-Martínez (2017), al analizar el efecto del estilo de aprendizaje predominante reflexivo con promedios más altos en las mujeres y asociado a la edad entre 26 y 30 años.

El cuestionario que se propone en la presente investigación permite identificar elementos de autocontrol en las actividades académicas de los estudiantes, de tal manera que pudieran relacionarse con aspectos individuales como el locus de control, como lo señala el análisis que realizan Mayora-Pernía y Fernández (2015), al referir que si el estudiante tiende a adoptar actitudes de conformismo y apatía (locus de control externo) su rendimiento académico disminuye. Con lo cual, se pudiera identificar la percepción que el estudiante tiene de su propia concentración, de su gusto por las tareas, de su participación en estudios extra-aula y de la expresión libre de sus ideas (componentes de la dimensión aportación en las actividades académicas del RAU) a fin de que futuras investigaciones la relacionen con este aspecto de la personalidad.

$\mathrm{Si}$ consideramos que la evaluación en el ámbito académicos permite confirmar el progreso del aprendizaje como interacción entre estudiantes, profesores, padres, empleadores y la comunidad (De Corte, 2015), el presente cuestionario facilitará identificar los componentes tanto subjetivos como objetivos del desempeño de los pupilos a nivel universitario. Es decir, estaría más cercano a valorar la acepción de "competencia" que involucra los componentes cognitivos. De tal manera que pudiera especificarse el grado de compromiso en las actividades asignadas por la institución y las generadas como parte de la autoregulación en estudiantes universitarios, acorde con el modelo cíclico de fases de Zimmerman (2013).

Una de las limitaciones de este estudio es que las escalas subjetivas del cuestionario RAU presentaron correlación débil con las actividades de estudio, posiblemente porque no es común que el estudiante registre el número de horas que dedica al estudio fuera del aula, el número de material de estudio (libros, artículos de lectura) o porque es limitada la producción académica a este nivel (publicaciones). Cabe mencionar que la fiabilidad de los factores del componente subjetivo fue de moderada a débil, seguramente debido a que los participantes conformaron muestras no probabilísticas, lo que implica ser cautelosos en las inferencias o generalizaciones poblacionales.

Finalmente, se enfatiza que el presente estudio es un análisis parcial del cuestionario y futuras investigaciones pudieran contribuir al análisis de validez de criterio, al relacionar el instrumento con otros componentes del proceso de aprendizaje, como la motivación o el compromiso, entre otros.

A pesar de las limitaciones expresadas, la presente investigación ofrece evidencias de fortalezas psicométricas para el cuestionario Rendimiento Académico Universitario que en futuras investigaciones $y$ en el ejercicio pedagógico, pudiera contribuir para el progreso del aprendizaje autorregulado por estudiantes universitarios.

\section{Conflicto de intereses}

Los autores declaran no tener ningún conflicto de intereses

\section{Financiamiento}

El presente estudio no recibió financiamiento para su desarrollo.

\section{Referencias}

Alducin-Ochoa, J .M., \& Vasquéz-Martínez, A. I. (2017). Estilos de aprendizaje, variables sociodemográficas y rendimiento académico en estudiantes de Ingeniería de Edificación. Revista Electrónica Educare, 21(1), 1-31. http://dx.doi.org/10.15359/ree.21-1.18

Andrés, A., Solanas, A., \& Salafranca, L. (2012). Interpersonal perception, personality, and academic achievement: A dyadic approach for the study of undergraduate performance. Anales de Psicología, 28(1), 97-106. http://www.redalyc.org/html/167/16723161012/ 
Aragón, M., \& Jiménez, Y. I. (2009). Diagnóstico de los estilos de aprendizaje en los estudiantes. CPU-e, Revista de Investigación Educativa $\quad 9, \quad 1(1), \quad 1-21$. http://www.redalyc.org/pdf/2831/2831217140 02.pdf

Ávalos, M. L., Ramírez, J. C., Oropeza, R., Palos, M. Ú., Miranda, R. B., \& Palomar, G. M. (2018). Bienestar escolar y rendimiento académico en estudiantes de bachillerato. Revista Internacional PEI, 6(14), 7-24. http://www.peiac.org/Revista/Numeros/No14/ indice.html

Avendaño, C., Gutiérrez, K., Salgado, C., \& Alonso, J. (2016). El rendimiento académico de los estudiantes la implementación del modelo de formación por competencias. Formación Universitaria, 9(3), 3-10. http://dx.doi.org/10.4067/S0718-

50062016000300002

Bolívar, J., \& Rojas, F. (2014). Estudio de la autopercepción y los estilos de aprendizaje como factores asociados al rendimiento académico en estudiantes universitarios. RED Revista de Educación a Distancia, 44(1), 1-13. http://www.um.es/ead/red/44/bolivar.pdf

Broc, M. A. (2011). Voluntad para estudiar, regulación del esfuerzo, gestión eficaz del tiempo y rendimiento académico en alumnos universitarios. Revista de Investigación Educativa, 29(1), 171-185.

http://revistas.um.es/rie/article/view/110731/1 26992

Caballero, C., Abello, R., \& Palacios, J. (2007). Relación del Burnout y el rendimiento académico con la satisfacción frente a los estudios en estudiantes universitarios. Avances en Psicología Latinoamericana. 25(2), 98-111.

http://www.redalyc.org/articulo.oa?id=79925207

Catena, A., Ramos, M., \& Trujillo, H. (2000). Análisis multivariado: Un manual para investigadores. Ed. Biblioteca Nueva.

Chacón, F., Padial, R., Yedra, S., Bretón, S., Cepero, M., \& Zurita, F. (2017). Relación entre rendimiento académico y autoconcepto en jugadoras de baloncesto de categoría cadete en competición nacional extraescolar. Revista Euroamericana de Ciencias del Deporte. 6(2), 75-80. https://digitum.um.es/xmlui/bitstream/10201/5 4237/1/300411-1029651-1-SM.pdf

De Corte, E. (2015). Aprendizaje constructivo, autorregulado, situado y colaborativo: Un acercamiento a la adquisición de la competencia adaptativa (matemática). Revista Páginas de Educación, 8(2), 69-87. http://revistas.ucu.edu.uy/index.php/paginasde educacion/article/view/690/678

Diaz, E. (2012). Estilos de Aprendizaje. Revista EÍDOS, 1(1), 5-10.

https://doi.org/10.29019/eidos.v0i5.88

Esguerra, G., \& Guerrero, P. (2010), Estilos de aprendizaje y rendimiento académico en estudiantes de Psicología. Revista Diversitas Perspectivas en Psicología, 6(1), 97-109. http://www.redalyc.org/pdf/679/67916261008 .pdf

García, R., Cuevas, O. Vales, J., \& Cruz, R. (2012). Impacto del Programa de Tutoría en el desempeño académico de los alumnos del Instituto Tecnológico de sonora. Revista Electrónica de Investigación Educativa, 14 (1), 106-121.

http://redie.uabc.mx/vol14no1/contenidogarciaetal.html

Gómez, P., Pérez, C., Parra, P., Ortiz, L., Matus, O., Mccoll, P., Torres, G., \& Meyer, A. (2015). Relación entre el bienestar y el rendimiento académico en alumnos de primer año de medicina. Revista Médica de Chile, 14(3), 930-937.

https://dx.doi.org/10.4067/S00349887201500 0700015

Gorostiaga, A. Balluerka, N, Ulacia, I., \& Aliri, J. (2018). Evaluación de la iniciativa personal en el ámbito educativo y su relación con la actitud emprendedora y el rendimiento académico. Revista Iberoamericana de Diagnóstico y Evaluación - e Avaliação Psicológica. 48(3), 105-116. https://doi.org/10.21865/RIDEP48.3.09

Ibarra, M. C., \& Michalus, J. C. (2010). Análisis del rendimiento académico mediante un modelo logit. Revista Ingeniería Industrial. $1(2), 47-56$.

http://revistas.ubiobio.cl/index.php/RI/article/ view/56

Inzunza, B, Pérez, C, Márquez, C., Ortiz, L., Marcellini, S., \& Duk, S. (2018). Estructura 
factorial y confiabilidad del Cuestionario de Motivación y Estrategias de Aprendizaje, MSLQ, en estudiantes universitarios Chilenos de Primer Año. Revista Iberoamericana de Diagnóstico y Evaluación - e Avaliação Psicológica. $\quad 47(2), \quad 21-35$. https://doi.org/10.21865/RIDEP47.2.02

Lamas H. (2015). Sobre el rendimiento Escolar. Propósitos y Representaciones, 3(1), 313-336. http://revistas.usil.edu.pe/index.php/pyr/article /view/74

Lizasoain, L. L., \& Joaristi, L. J. (2010). Estudio diferencial del rendimiento académico en lengua española de estudiantes de educación secundaria de baja california (México). Revista Iberoamericana de Evaluación Educativa, 3(3), 115-134. http://www.rinace.net/riee/numeros/vol3num3/art6.pdf

Lloret-Segura, S., Ferreres-Traver, A., HernándezBaeza, A., \& Tomás-Marco, I. (2014). El análisis factorial exploratorio de los ítems: Una guía práctica, revisada y actualizada. Anales de Psicología, 30(3), 1151-1169, http://www.redalyc.org/pdf/167/16731690031 .pdf

Mayora-Pernía, C., \& Fernández, N. (2015). Locus de control y rendimiento académico en educación universitaria: Una revisión bibliográfica. Revista Electrónica Educare, 19(3), 1-23. http://dx.doi.org/10.15359/ree.193.16

Molina, M. L. (2015). Valoración de los criterios referentes al rendimiento académico y variables que lo puedan afectar. Revista Médica Electrónica, 37(6), 617-626. http://scielo.sld.cu/scielo.php?script=sci_artte $\mathrm{xt} \&$ pid $=$ S1684-18242015000600007

Núñez, F. C., \& Quiñonez, A. (2012). Autoestima y rendimiento académico en estudiantes de enfermería de Poza Rica, Veracruz, México. Uni-pluir/versidad, 12(1), 25-35. https://aprendeenlinea.udea.edu.co/revistas/in dex.php/unip/article/view/13275

Ortiz-Fernández, L., et al. (2015), Estrategias, estilos de aprendizaje y rendimiento académico en estudiantes ingresantes de Odontología. Odontología Sanmarquina, 17(2), 76-81. http://dx.doi.org/10.15381/os.v17i2.11050
Panadero, E., \& Alonso, J. (2014). ¿Cómo autorregulan nuestros alumnos? Revisión del modelo cíclico de Zimmerman sobre autorregulación del aprendizaje. Anales de Psicología. 30(2), 450-462.

http://revistas.um.es/analesps/article/viewFile/ analesps.30.2.167221/161091

Reyes, R., Godínez, F., Ariza, F. J., Sánchez, F., \& Torreblanca, O. F. (2016). Un modelo empírico para explicar el desempeño académico de estudiantes de bachillerato. Perfiles Educativos, 36(146), 45-62. https://www.sciencedirect.com/science/article/ pii/S0185269814701278

Ruiz, M. A., Pardo, A., \& San Martín, R. (2010). Modelos de ecuaciones estructurales. Papeles del Psicólogo, 31(1), 34-45. Recuperado de http://www.papelesdelpsicologo.es/pdf/1794.p df

Ruslin, A., \& Jelas, Z. M. (2010). Teaching and learning styles in Higher Education institutions: Do they match? Procedia-Social and Behavioral Sciences, 7(1), 680-684. https://doi.org/10.1016/j.sbspro.2010.10.092

Salum-Fares, A., Aguilar, R., \& Reyes, C. (2011). Autoconcepto y rendimiento académico en estudiantes de escuelas secundarias públicas y privadas de Ciudad Victoria, Tamaulipas, México. Revista Internacional de Ciencias Sociales y Humanidades, SOCIOTAM, 14(2), 255-272.

http://www.redalyc.org/pdf/654/65421407010 .pdf

Zimmerman, B. J. (2013). From cognitive modeling to self-regulation: A social cognitive career path. Educational Psychologist, $\quad 48(3), \quad$ 135-147. https://doi.org/10.1080/00461520.2013.794676 